OPEN ACCESS

Edited by:

Fang Pan,

Shandong University, China

Reviewed by:

Apiwat Mutirangura,

Chulalongkorn University, Thailand

Antonius Plagge,

University of Liverpool,

United Kingdom

*Correspondence:

Yuan-Xiang Tao

yuanxiang.tao@njms.rutgers.edu

Yanqiu $A_{i}$

aiyanqiu82@163.com

tThese authors have contributed equally to this work

Specialty section:

This article was submitted to

Epigenomics and Epigenetics,

a section of the journal

Frontiers in Cell and Developmental

Biology

Received: 13 May 2020

Accepted: 31 July 2020

Published: 28 August 2020

Citation:

Li M, Su S, Cai W, Cao J, Miao X,

Zang W, Gao S, XU Y, Yang J, Tao Y-X and $\mathrm{Ai} Y$ (2020) Differentially

Expressed Genes in the Brain of Aging Mice With Cognitive Alteration and Depressionand Anxiety-Like Behaviors Front. Cell Dev. Biol. 8:814.

doi: 10.3389/fcell.2020.00814

\section{Differentially Expressed Genes in the Brain of Aging Mice With Cognitive Alteration and Depression- and Anxiety-Like Behaviors}

\author{
Mengqi Li1,2†, Songxue Su ${ }^{2,3+}$, Weihua $\mathrm{Cai}^{2,3}$, Jing $\mathrm{CaO}^{2,3}$, Xuerong Miao ${ }^{4}$, \\ Weidong Zang ${ }^{2,3}$, Shichao $\mathrm{Gao}^{5}$, Ying $\mathrm{Xu}^{5}$, Jianjun Yang ${ }^{1,2}$, Yuan-Xiang Tao ${ }^{6 *}$ and \\ Yanqiu $\mathrm{Ai}^{1,2 *}$
}

\begin{abstract}
' Department of Anesthesiology, Pain and Perioperative Medicine, The First Affiliated Hospital of Zhengzhou University, Zhengzhou, China, ${ }^{2}$ Neuroscience Research Institute, Zhengzhou University Academy of Medical Sciences, Zhengzhou, China, ${ }^{3}$ Department of Anatomy, College of Basic Medicine, Zhengzhou University, Zhengzhou, China, ${ }^{4}$ Department of Anesthesiology and Intensive Care, Third Affiliated Hospital of Second Military Medical University, Shanghai, China, ${ }^{5}$ Department of Pharmaceutical Sciences, School of Pharmacy and Pharmaceutical Sciences, The State University of New York, Buffalo, NY, United States, ${ }^{6}$ Department of Anesthesiology, New Jersey Medical School, Rutgers, The State University of New Jersey, Newark, NJ, United States
\end{abstract}

Despite the great increase in human lifespan with improved medical care, the physiological and pathological changes such as memory and cognitive disorders and associated anxiety and depression are major concern with aging. Molecular mechanisms underlying these changes are little known. The present study examined the differentially expressed genes (DEGs) and the genes with differentially expressed isoforms in three brain regions, anterior cingulate cortex (ACC), amygdala and hippocampus, throughout the lifespan of mice. Compared to 2-month old mice, both 12- and 24-month old mice displayed memory and cognitive impairments in the Morris water maze, Y-maze, and novel object recognition tests and depression- and anxiety-like behaviors in the tail suspension, forced swimming, open field, and elevated plus maze tests. RNA sequencing analysis identified 634 and 1078 DEGs in ACC, 453 and 1015 DEGs in the amygdala and 884 and 1054 DEGs in hippocampus in the 12- and 24-month old mice, respectively. Similarly, many genes with differentially expressed isoforms were also identified in these three brain regions in the 12- and 24-month old mice. Further functional analysis revealed that many DEGs and the genes with differentially expressed isoforms in the ACC and amygdala were mapped to depression- and anxiety-related genes, respectively and that a lot of DEGs and the genes with differentially expressed isoforms in hippocampus were mapped to cognitive dysfunction-related genes from both 12- and 24-month old mice. All of these mapped DEGs and the genes with differentially expressed isoforms were closely related to neuroinflammation. Our findings indicate that these neuroinflammation-related DEGs and the genes with differentially expressed isoforms are likely new targets in the management of memory/cognitive impairment and emotional disorders during the aging.

Keywords: cognitive dysfunction, depression, anxiety, RNA sequencing, aging mice 


\section{INTRODUCTION}

With rapid socio-economic development, the life expectancy of human being is increasing (Beard et al., 2016). However, agerelated disorders such as Alzheimer's disease (AD) and dementia afflict vast majority of aged patients and become most significant public health issues and considerable challenges around the world (Kanasi et al., 2016). Indeed, the aging process may involve a series of behavioral and psychological dysfunction due to neurodegenerative changes in cell homeostasis and biological pathways (Gurkar and Niedernhofer, 2015). Clinical investigation indicates that aged patients with dementia often present clusters of behavioral symptoms such as memory and cognitive disorder, depression, anxiety, and neuropathic pain (Frisoni et al., 1999; Herrup, 2010; Salthouse, 2012; Harada et al., 2013). Although cognitive and functional impairment as the hallmark of aging is often emphasized, neuropsychiatric symptoms are also directly responsible for the reduced quality of life and the increased rates of disability in patients and their families. Current treatments for these age-related disorders are unsatisfactory and/or produce adverse effects at least in part due to incomplete understanding of molecular mechanisms underlying these disorders. Therefore, identifying the differentially expressed genes (DEGs) in the cognitive impairment-, depression-, or anxiety-associated brain regions of aging mice is a key step for searching new targets for novel treatments and preventative tactics for the age-related disorders.

Several brain regions such as hippocampus, amygdala and anterior cingulated cortex (ACC) are involved in the aging process and participate in the pathological processes of age-related cognitive and emotional disorders (Killgore and Yurgelun-Todd, 2004; Etkin et al., 2006; Liu W. et al., 2017; Martin et al., 2017; Ewbank et al., 2018; Piel et al., 2018). Indeed, the gene and protein networks among these three brain regions (Killgore and Yurgelun-Todd, 2004; Etkin et al., 2006; Ewbank et al., 2018; Piel et al., 2018) directly regulate cognitive dysfunction and depression/anxiety-like behaviors (Chao et al., 2016; Liu W. et al., 2017; Martin et al., 2017; Scalici et al., 2017; Yan et al., 2018). Although there are numerous hypotheses for understanding the pathogenesis of age-related memory deficits and emotional dysfunction, the dysregulation of genes and proteins in brain regions during aging has been widely acknowledged (Zhang et al., 2016; Alam et al., 2018; Holmes et al., 2018; Mehta et al., 2018). Several studies have identified DEGs between young- and aged-mice, but most of them focused on only one brain region and/or at a one-aged time point (Reichwald et al., 2009; Cribbs et al., 2012). Thus, it is imperative to obtain more thorough molecular profiles with aging by examining different gene changes in multiple brain sites throughout multiple time points during the lifespan.

To better understand the network of gene changes in the ACC, hippocampus and amygdala with aging, the present study focused on gene expression that changed in these three brain regions throughout the lifespan of mice. To this end, we employed the 2-, 12-, and 24-month old mice and first observed memory and cognitive performance, depression- and anxietylike behaviors and the responses to nociceptive stimulation. We then collected ACC, amygdala and hippocampus from these mice and performed next-generation RNA sequencing with a higher sequencing depth and without mRNA poly-A tail selection. Finally, we analyzed and compared the transcriptome profiles in these regions among these different age mice. Our findings may provide novel information that may be used to identify new targets for the prevention and treatment of age-related neurodegenerative disorders.

\section{MATERIALS AND METHODS}

\section{Animals}

The 7-week old C57BL/6J wild-type male mice were obtained from Charles River Laboratory (Beijing, China) and SinoBritish SIPPR/BK Lab (Shanghai, China) in this study. Animals were housed in a temperature-controlled room and exposed to a standard $12 \mathrm{~h}$ light-dark cycle and normal illumination environment. Three groups of different age mice (2-, 12-, and 24-month old; 8 mice/group) were used. The animals had at least 1 week for acclimation to facility environment before behavioral tests, which were carried out in a quiet room between 10:00 a.m. and 4:00 p.m. (daytime). The mice were acclimated to experimental apparatus for $45 \mathrm{~min}$ before the tests. All behavioral tests were done within 1 week. All procedures were conducted in accordance with the ethical guidelines of the National Institutes of Health and the International Association for the Study of Pain and approved by the Animal Care and Use Committee of Zhengzhou University. All efforts were made to minimize animal suffering and to reduce the number of animals used.

\section{Open Field Test}

The open field test (OFT) was performed to evaluate locomotion activity and anxiety-like behavior as described previously (Sun et al., 2016). The open field apparatus consisted of a gray Plexiglas box (50-cm long $\times 50-\mathrm{cm}$ wide $\times 40-\mathrm{cm}$ high), which was divided into 16 virtual squares (12 peripheral squares and 4 central squares). The light source was $1.8 \mathrm{~m}$ above the ground. The light intensity was $50 \mathrm{~W}$. Briefly, mice were allowed to explore the arena for $5 \mathrm{~min}$ freely. Time spent in the central squares, distance in the central squares, numbers of entries, and the total distance traveled were recorded and analyzed by the video tracking system of SMART 3.0 (Panlab Harvard Apparatus, Spain). After each test, the open-field arena was cleaned with $10 \%$ bleach and 75\% ethanol (Davalli et al., 2016; Fougere et al., 2017; Hekmatimoghaddam et al., 2017; Liu E. et al., 2017; CostaFerreira et al., 2019; Zhou et al., 2019).

\section{Elevated Plus-Maze Test}

The elevated plus-maze (EPM) test was used for assessing anxiety-like behavior (Rice et al., 2019). An elevated plus-shaped maze (50 $\mathrm{cm}$ above the floor) consisted of a central platform (5$\mathrm{cm}$ long $\times 5$-cm wide), two open arms (30-cm long $\times 5$ - $\mathrm{cm}$ wide), and two closed arms (30-cm long $\times 5$ - $\mathrm{cm}$ wide $\times 15-\mathrm{cm}$ high). The open and close arms were situated opposite to each other. In the testing session, each mouse was placed in the central platform facing one of the open arms and allowed to explore freely for 
$5 \mathrm{~min}$ in a dim room. The percentage of open arm entries was calculated as the number of open-arms entries divided by total open and closed arms entries. The percentage of time spent in open arms was calculated as time spent in the open arms divided by total time (Franco et al., 2017; Klinger et al., 2019).

\section{Forced Swimming and Tail Suspension Tests}

Forced swimming and tail suspension tests (FST and TST) were used to measure depression-like behaviors by recording the immobility time of animals. In the FST (Inestrosa and Varela-Nallar, 2014), the mouse was placed individually into the transparent beakers $(20-\mathrm{cm}$ diameter by $25-\mathrm{cm}$ height) for $6 \mathrm{~min}$ with a water depth of $15 \mathrm{~cm}$ at $24-25^{\circ} \mathrm{C}$. The duration of cumulative immobility was recorded during the last $4 \mathrm{~min}$ within the 6-min testing period. The immobility was identified as the mouse floated in the water without struggling. For the tail suspension testing (Wadhwa et al., 2018), mice were suspended through the taped and affixed approximately $1 \mathrm{~cm}$ from the tip of the tail, $50 \mathrm{~cm}$ above the floor. All mice were suspended for $6 \mathrm{~min}$ in each session, and the immobility period was recorded in the last $4 \mathrm{~min}$ of the testing period. Mice were considered to be immobile only when they were passive and completely stationary. The experimental procedures were recorded by digital video-camera.

\section{Novel-Object Recognition Test}

The novel-object recognition (NOR) test was conducted in a square gray Plexiglas test box $(50-\mathrm{cm}$ long $\times 50$ - $\mathrm{cm}$ wide $\times 40$ $\mathrm{cm}$ high) to test learning and memory (Lueptow, 2017). Animals were habituated to the arena for $5 \mathrm{~min}$ every day for 2 days before the test. Each mouse was allowed to explore two identical objects $(\mathrm{A} 1+\mathrm{A} 2)$ placed in the arena for $5 \mathrm{~min}$. Three trials were conducted with an inter-trial interval of $15 \mathrm{~min}$. Animals were kept in cages until the next trial at the intervals. Recognition index from the third trial during training session was used to rule out mouse preference for two objects. Mice were put back to the center of the arena and explore for another 5 min with a novel object (B; replacing A2) at 3 and $24 \mathrm{~h}$ after the third trial. Watching, licking or touching the object with forepaws and facing the object at the distance of approximately $2 \mathrm{~cm}$ were considered as object exploration. Behaviors were recorded and analyzed by the video tracking system of Smart 3.0 (Panlab Harvard Apparatus, Spain). Recognition index was calculated as the investigation time novel object / (investigation time novel object + investigation time familiar object) (Lueptow, 2017; Costa-Ferreira et al., 2019; Zhou et al., 2019).

\section{Y-Maze Spontaneous Alternation Test}

Spatial working memory was tested in the Y-maze spontaneous alternation task (Liu E. et al., 2017). Spontaneous alternation performance was assessed by a gray Plexiglas 3-arm Y-maze (each arm: $30-\mathrm{cm}$ long $\times 7-\mathrm{cm}$ wide $\times 15-\mathrm{cm}$ high) under weak light conditions. Mice were randomly placed in one of the three arms and allowed to explore freely for $5 \mathrm{~min}$. The number and the sequence of the visited arms were recorded.
The number of arm entries and the percentage of spontaneous alternation [number of alternation / (number of total arm entries2) $\times 100 \%$ ] was calculated.

\section{Morris Water Maze Test}

Morris water maze (MWM) was another approach to evaluate spatial learning in rodents, as described previously (Vorhees and Williams, 2006; Xu et al., 2018). Mice were trained in a water maze $(120 \mathrm{~cm}$ in diameter, $50 \mathrm{~cm}$ in depth) filled with water (25 $\mathrm{cm}$ in depth, $23 \pm 0.5^{\circ} \mathrm{C}$ ). The pool was divided into four virtual quadrants, each with a cue to assist the mice to find the hidden platform $(8.5 \mathrm{~cm}$ in diameter, $24 \mathrm{~cm}$ in height) that was submerged $1 \mathrm{~cm}$ under the water in the middle of one of the four quadrants. Each mouse was placed in a quadrant other than where the hidden platform was located, facing the wall of the pool, and was given $60 \mathrm{~s}$ to find the platform and $15 \mathrm{~s}$ to stay on it. Animals that did not find the platform were gently guided and placed on it during the learning test. Mice were trained for five consecutive days (three trials per day with an inter-trial interval of $20 \mathrm{~min}$ ), and mean latency (time taken for the mouse to find the platform) from three trails per day was calculated. At 1 or $24 \mathrm{~h}$ after the training, the hidden platform was removed and learning was assessed through measuring the latency taken for the mouse to find the place where the hidden platform was located before and the number of crossing this place within 60 s (Klinger et al., 2019; Zhou et al., 2019).

\section{Mechanical Test}

Paw withdrawal frequencies in response to mechanical stimuli were tested as described before (Xu et al., 2018). Briefly, each mouse was placed individually in a Plexiglas chamber on an elevated mesh. Two calibrated von Frey filaments $(0.07 \mathrm{~g}$ and $0.4 \mathrm{~g}$; Stoelting, Kiel, WI, United States) were applied to the hind paw for approximately $1 \mathrm{~s}$, respectively. Each stimulation was repeated ten times to both hind paws. The occurrence of paw withdrawal in each of these ten trials was expressed as a percent response frequency [(number of paw withdrawals/10 trials) $\times 100=\%$ response frequency].

\section{Thermal Test}

Paw withdrawal latencies to noxious thermal stimulation were examined as previously described (Porsolt et al., 1978). Each mouse was placed in a Plexiglas chamber on a glass plate. Radiant heat from the Plantar Test Instrument (Ugo Basile 37370, Italy) was applied by aiming a beam of light through the glass plate to the middle of the plantar surface of each hind paw. When the mouse lifted its foot, the light beam was cut off. The length of time between the start of the light beam and the foot lift was defined as the paw withdrawal latency. Each trial was repeated five times at 5-min intervals for each side. A cut-off time of $20 \mathrm{~s}$ was used to avoid tissue damage to the hind paw.

\section{RNA Extraction}

On the final day, the mice were sacrificed immediately after behavioral tests. The anterior cingulate cortex (ACC), hippocampus and amygdala were collected in the tubes 
containing RNAlater (Ambion, Austin, TX, United States). Total RNA was extracted using the miRNeasy kit with oncolumn digestion of genomic DNA (QIAGEN, Valencia, CA, United States). RNA concentration was measured using the NanoDrop 2000 Spectrophotometer (Thermo Figher Scientific, Wilmington, DE, United States). The ratios of A260/280 nm were between 1.97 and 2.08 in all samples. RNA integrity was assessed using RNA Nanochips in an Agilent 2100 Bioanalyzer (Agilent Technologies, Santa Clara, CA, United States). RNA integrity number (RIN) was between 7.5 and 8.4.

\section{RNA Sequencing}

Three biological replicates (three mice) from each age group were used in the RNA-seq analysis. Total RNA extracted above was subjected to rRNA depletion by Ribo-Zero rRNA Removal (Human/Mouse/Rat) Kit (Illumina, San Diego, CA, United States). Strand-specific RNA libraries were prepared using TruSeq Stranded Total RNA Sample Preparation Kit (Illumina) without poly-A selection. All assays were performed according to the manufacturer's instructions. Sequencing was carried out at the Illumina Nova6000 plate High Output Model (Illumina, San Diego, CA, United States) (Hrdlickova et al., 2017), in a $2 \times 150$ bp paired-end configuration, with a total of more than 2,666 $\mathrm{M}$ reads per lane (at least $40 \mathrm{M}$ reads per sample). All sequencing data are available in NCBI database (accession number: SRP271007).

\section{Bioinformatics Analysis}

Nine samples from ACC, hippocampus, and amygdala (three repeats/region) were subjected to multiplexing, sequencing, differential gene expression, and transcript expression analysis. Briefly, the sequences were first trimmed in quality using trimmomatic-0.32 (Minimal length $50 \mathrm{bp}$, leading and trailing Phred Q 30). The resulting sequencing data were then mapped to the musculus genome sequence version GRCm38.72, downloaded from ENSEMBLE. Gene hit counts and reads per kilobase per million mapped reads (RPKM) were calculated for each gene to determine expression levels. DEGs and the genes with differentially expressed isoforms were filtered to $P$-value 0.05 and $\log$ base twofold change $(\mathrm{LFC}) \geq 1$ or $\leq-1$. The above RNA-Seq analyses, including mapping, read counts and expression analysis, were carried out within the CLCbio software environment (CLC Genomics Workbench 7.0.2, CLC genomics Server). Mapped reads were visualized on the UCSC browser using bigwig files converted from bam files. The heatmap was generated using Heatmaper ${ }^{1}$. To analyze the functions of DEGs and the genes with differentially expressed isoforms, we used GeneCards database $^{2}$ and CTD database ${ }^{3}$ to map them with depression and anxiety in the ACC and amygdala as well as with cognitive dysfunction in hippocampus. They were also mapped to inflammation, apoptosis, oxidative stress, synaptic plasticity, glutamate-receptor pathway, and DNA methylation.

\footnotetext{
${ }^{1}$ http://www.heatmapper.ca/expression/

${ }^{2}$ https://www.genecards.org/

${ }^{3}$ http://ctdbase.org/
}

\section{Quantitative Real-Time RT-PCR}

Total RNA was reverse-transcribed using the ThermoScript reverse transcriptase (Invitrogen) according to the manufacturer's instructions with the oligo (dT) primers. cDNA was amplified by real-time PCR using the primers listed in Supplementary Table 1 (Integrated DNA Technologies, Coralville, IA, United States). Each sample was run in triplicate in a $20 \mu \mathrm{l}$ reaction with $250 \mathrm{nM}$ forward and reverse primers and $10 \mu \mathrm{l}$ of Advanced Universal SYBR Green Supermix (Bio-Rad Laboratories, Hercules, CA, United States). Reactions were performed in a BIO-RAD CFX96 real-time PCR system. The cycle parameters were set as follows: an initial $3 \mathrm{~min}$ incubation at $95^{\circ} \mathrm{C}$, followed by 40 cycles of $95^{\circ} \mathrm{C}$ for $10 \mathrm{~s}, 60^{\circ} \mathrm{C}$ for $30 \mathrm{~s}$, and $72^{\circ} \mathrm{C}$ for $30 \mathrm{~s}$. Ratios of mRNA levels from 12- or 24-month old mice to mRNA levels from 2-month old mice were calculated using the $\Delta \mathrm{Ct}$ method $(2-\Delta \Delta \mathrm{Ct})$ at a threshold of 0.02 . All data were normalized to an internal housekeeping gene Gapdh (Wen et al., 2020).

\section{Functional Enrichment Analysis of Differentially Expressed Genes}

For biological pathway analysis, approximately 634, 453, and 884 DEGs respectively from ACC, amygdala, and hippocampus of the 12-month old mice and 1078, 1015, and 1054 DEGs, respectively from ACC, amygdala, and hippocampus of the 24month old mice were selected based on significantly differential changes in their expression as compared with the 2-month old mice. Panther Classification System database system was used to categorize the differential expression genes. (C) 2015, Paul Thomas ${ }^{4}$ ). To comprehensively analyze the functions of the DEGs in the ACC, amygdala and hippocampus, all DEGs (including the upregulated genes and downregulated genes) were analyzed using the gene ontology enrichment analysis from the Database for Annotation, Visualization, and Integrated Discovery $\left(\right.$ DAVID $\left.^{5}\right)$.

\section{Protein-Protein Interaction Network Construction}

STRING (version: 11.0) database ${ }^{6}$ was used to predict the interaction between the proteins encoded by the DEGs. The protein-protein interaction (PPI) network was built using Cytoscape software (version: 3.6.07) (Liu et al., 2019). The connection degree of each node was calculated through the centiscape plugin, and the first 50 genes that were selected based on connection degree were used to construct networks. Nodes were excluded from the network due to interaction with other nodes. The size of the node was determined by the degree of connection degree in the network, and use red, blue, and purple colors to mark the genes related to inflammation, apoptosis, and both inflammation and apoptosis, respectively.

\footnotetext{
${ }^{4}$ pantherdb.org

${ }^{5}$ http://david.abcc.ncifcrf.gov/

${ }^{6}$ https://string-db.org/cgi/

${ }^{7}$ www.cytoscape.org/
} 


\section{Correlational Analysis Between mRNA Levels of DEGs and Mouse Behaviors}

Three DEGs were selected from ACC, amygdala and hippocampus, respectively, of 2- and 12-month old mice according to their scores between the genes and depression, anxiety and cognitive dysfunction based on the GeneCards database $^{2}$ and CTD database ${ }^{3}$. A correlation analysis between the expressional levels of these DEGs from 2- and 12-month old mice and behavioral scores from these corresponding mice was performed via the Pearson's method using the SPSS Software (IBM SPSS Statistics 21.0).

\section{Statistical Analysis}

All data were collected randomly and expressed as mean \pm SEM. The data were statistically analyzed with two-tailed, unpaired Student's $t$-test or one- or two-way ANOVA with repeated measures. When ANOVA showed a significant difference, the pairwise comparison between means was tested by the post hoc Tukey method. $P$-values less than 0.05 were considered statistically significant.

\section{RESULTS}

\section{General Appearance and Behaviors in Aging Mice}

As shown in Supplementary Figure 1, the body weights of 12 - and 24 -month old mice were increased by 1.24 -fold and 1.32 -fold, respectively, as compared with 2-month old mice. Additionally, there were differences in the general appearance among these three groups of mice. The 12- and 24-month old mice exhibited some aging appearances, including slight body bending with hair loss and lacking luster. The 24month old mice displayed lightly unstable gait and reduced spontaneous activity. There were no visible differences in the gait and spontaneous activity between the 12- and 2-month old mice.

\section{Impaired Cognitive and Memory Abilities in Aging Mice}

Cognitive and memory abilities in three groups of mice were observed by novel object recognition (NOR), Y-maze spontaneous alternation, and MWM tests. In the NOR test, the mice from 2-, 12-, and 24-month old did not exhibit any differences in basal precognitive behavior in the training session (Figure 1A). However, 3 and $24 \mathrm{~h}$ later after training, 12- and 24-month old mice displayed a significantly reduced recognition in exploring new objects as compared with the 2month old mice. Consistently, the Y-maze test showed that the percentages of spontaneous alternation were robustly decreased in 12- and 24-month old mice compared with those of 2month old mice (Figure 1B), indicating the decline in spatial memory. In addition, a significant reduction in the total number of arm entries was found in 24-month old mice, but not in 12-month old mice (Figure 1C). This indicates that 24-month old mice may have abnormal locomotor activity.
In the MWM test, 12-month old mice displayed a longer latency to reach the platform compared to 2-month old mice in the 5th trial in the learning curve (Figure 1D). Spatial and working memory was further determined by removing the platform. As shown in Figures 1E-H, 12month old mice exhibited the significantly lower numbers of crossing the place where the escape platform was located previously and the prolonged escape latencies at 1 and $24 \mathrm{~h}$ after training, suggesting the worse memory retention in 12-month old mice. As expected, we found that the total distances traveled at 1 and $24 \mathrm{~h}$ probe trials did not differ between 2- and 12-month old mice (Figures 1I,J), indicating that the performance differences observed above did not result from the changes in overall activity. The 24-month old mice were not employed in the MWM test considering the high risk of this test due to the reduced spontaneous activity and impaired locomotor function (see below) in these mice.

\section{Depression- and Anxiety-Like Behaviors in Aging Mice}

Forced swimming tests and tail suspension tests are the classical methods to screen the rapid behavioral changes in stressful situations. Immobility time was used as an indicator of depression in these tests. As shown in Figures 2A,B, immobility times in both 12 - and 24-month old mice were significantly prolonged as compared with 2-month old mice, demonstrating that the aging mice were more likely to give up struggling in both tests due to despair.

To determine the anxiety-like behavior in aging mice, we performed the open field and EPM tests. In the OFT, we found that the overall distance traveled was conspicuously decreased in 24-month old mice, but not in 12-month old mice (Figure 2C), suggesting the impaired locomotor activity in 24-month old mice. Further observations showed the reduced exploration activity in 12- and 24-month old mice evidenced by the considerable reductions in distance traveled and time spent in the center area (Figures 2D,E), as well as in numbers of entering the central area (Figure 2F). Similar to whatever we observed in OFT, the EPM test showed that both the percentage of time spent in the open arms (Figure 2G) and the percentage of open arm entries were much lower in 12- and 24-month old mice than those in 2-month old mice (Figures 2G,H). As expected, the significantly decreased number of total entries to both open and closed arms in 24-month old mice, but not 12month old mice, was seen (Figure 2I), further demonstrating impaired motor function only in 24-month old mice. Taken together, these findings indicate the depression- and anxiety-like behaviors in aging mice.

\section{Basal Nociceptive Behavioral Responses in Aging Mice}

To understand comprehensive phenotypes with aging, we examined basal nociceptive behavioral responses to mechanical and thermal stimuli in these three different ages of mice. As 


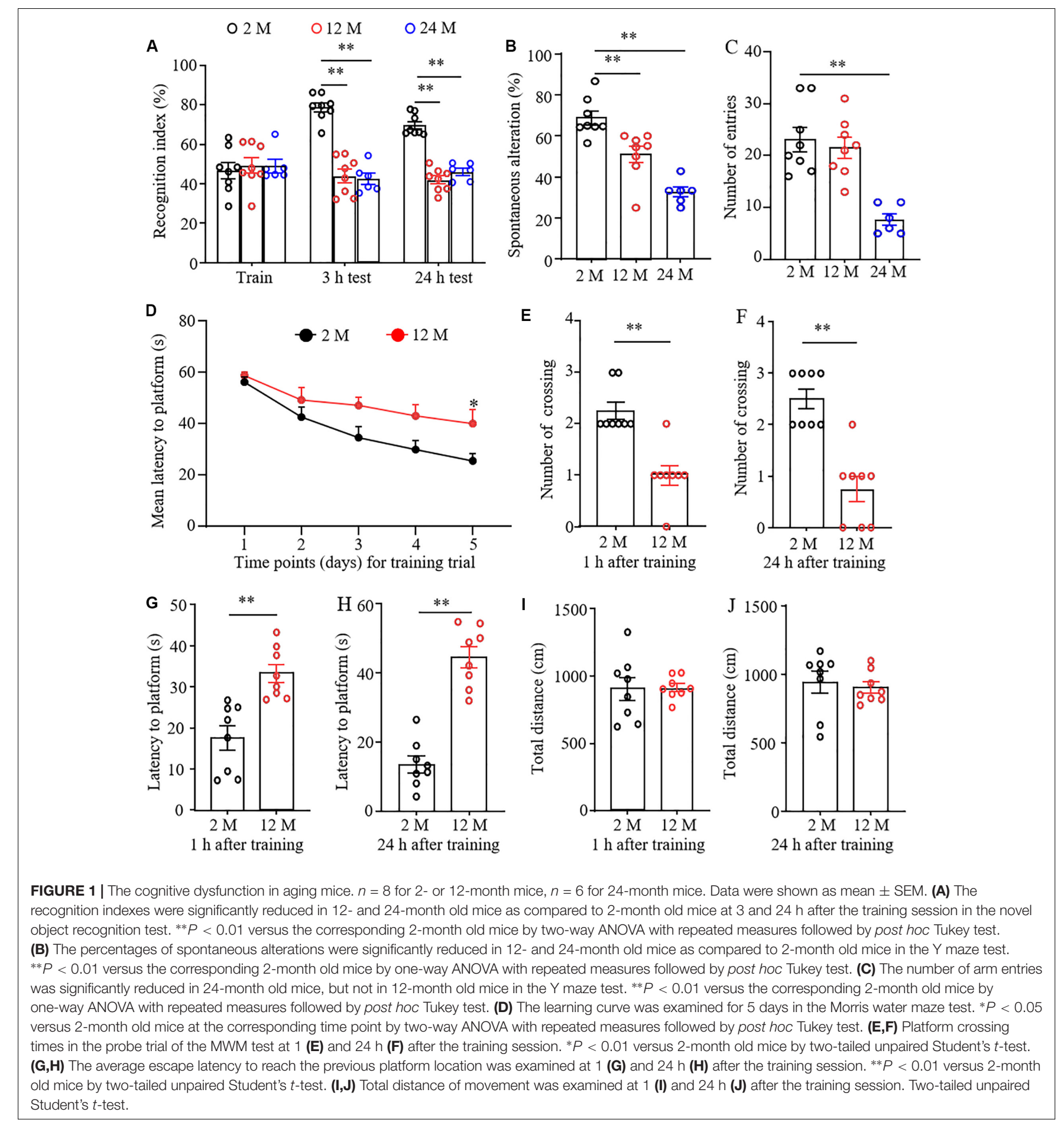

shown in Figure 3A-D, there were no significant differences in paw withdrawal frequencies in response to $0.07 \mathrm{~g}$ and $0.4 \mathrm{~g}$ von Frey filament stimuli among 2-, 12-, and 24-month old mice in both hind paws, although 24-month old mice exhibited a decreased tendency in response to $0.07 \mathrm{~g}$ von Frey filament stimulation. In addition, the mice from these three different ages showed similar paw withdrawal latencies to heat stimulation on both sides (Figures 3E,F).

\section{RNA-seq and Genome-Wide Read Mapping in ACC, Hippocampus, and Amygdala of Aging Mice}

To examine whether behavioral changes observed above are associated with DEGs in brain regions of aging mice, we carried out the RNA-sequencing analysis to define the gene expression profiles in the ACC, hippocampus, and amygdala from these 

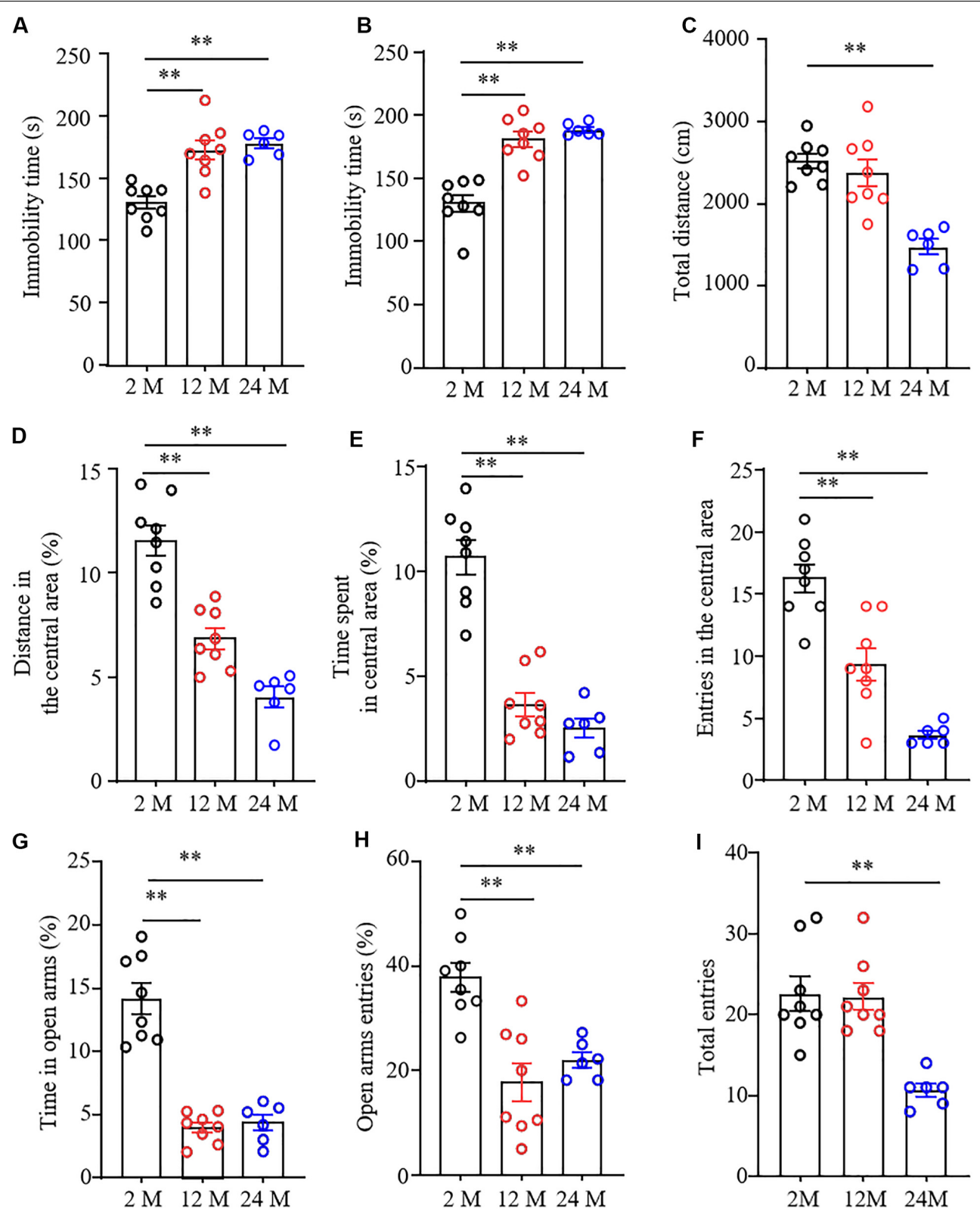

FIGURE 2 | Depression- and anxiety-like behavior in aging mice. $n=8$ for 2 - or 12 -month mice, $n=6$ for 24 -month mice. Data were shown as mean \pm SEM. (A,B) The immobility time was significantly increased in 12- and 24-month old mice in the forced swimming test (FST) (A) and tail suspension test (TST) (B) as compared to 2 -month old mice. ${ }^{* *} P<0.01$ versus 2 -month old mice by one-way ANOVA with repeated measures followed by post hoc Tukey test. (C) The total distance of 24-month-old mice in the open field test was less than that of 2 -month-old mice. ${ }^{* *} P<0.01$ versus 2 -month old mice by one-way ANOVA with repeated measures followed by post hoc Tukey test. (D-F) The percentage of distance in the central area (D), the time spent in the central area (E), and the entries in the central area (F) were significantly reduced in 12- and 24-month old mice in the open field test as compared to 2-month-old mice. ${ }^{* *} P<0.01$ versus 2 -month old mice by one-way ANOVA with repeated measures followed by post hoc Tukey test. (G,H) The percentages of the time spent in open arms (G) and open arms entries $\mathbf{( H )}$ were both significantly reduced in 12- and 24-month old mice as compared to 2-month old mice in the elevated plus maze test. ** $P<0.01$ versus 2 -month old mice by one-way ANOVA with repeated measures followed by post hoc Tukey test. (I) The total arm entries of 24-month old mice were less than those of 2-month old mice. **P $<0.01$ versus 2-month old mice by one-way ANOVA with repeated measures followed by post hoc Tukey test.

three different ages of mice. In order to visualize the collected data of DEGs, we generated the clustered heatmaps to show the changes in the genes by color intensity. As shown in Figure 4, compared to the gene expression profile from 2-month old mice, about 634 (510 increased and 124 decreased) and 1078
(805 increased and 273 decreased) DEGs in ACC, 453 (283 increased and 170 decreased) and 1015 (646 increased and 369 decreased) DEGs in the amygdala and 884 (485 increased and 399 decreased), and 1054 (759 increased and 295 decreased) DEGs in hippocampus were detected in 12- and 24-month old 

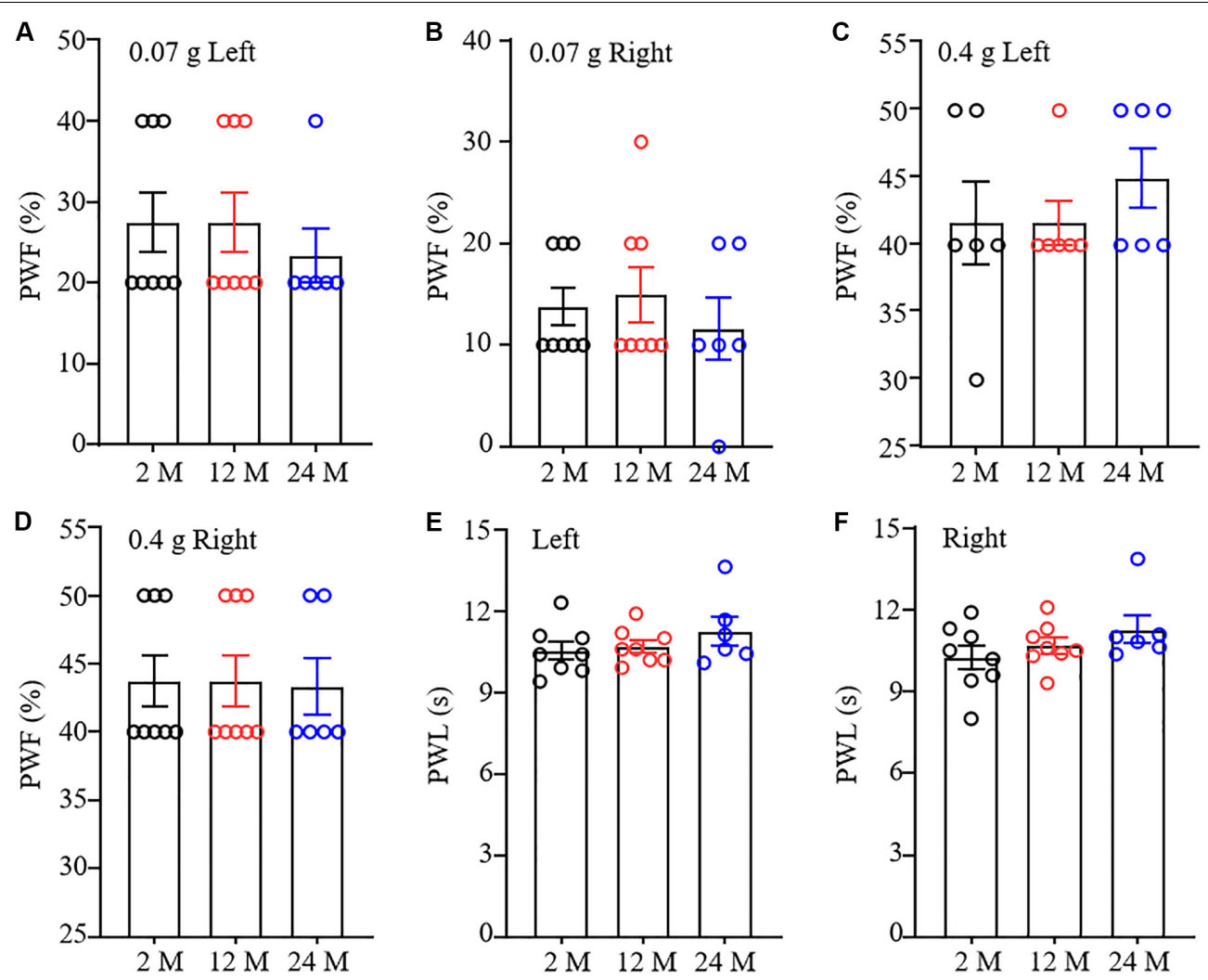

FIGURE 3 | Nociceptive response in different ages of mice. Paw withdrawal frequencies (PWF) in response to $0.07 \mathrm{~g}$ (A,B) and $0.4 \mathrm{~g}$ (C,D) von Frey filaments and paw withdrawal latency (PWL) on response to heat stimulation (E,F) on the left $(\mathbf{A}, \mathbf{C}, \mathbf{E})$ and right $(\mathbf{B}, \mathbf{D}, \mathbf{F})$ sides. $n=8$ for $2-$ or $12-$ month mice, $n=6$ for $24-$ month mice. Data were shown as mean \pm SEM. One-way ANOVA with repeated measures followed by post hoc Tukey test.

mice, respectively. Further analysis revealed that approximately 222 (187 increased and 35 decreased) DEGs in ACC, 171 (125 increased and 46 decreased) DEGs in the amygdala and 315 (229 increased and 86 decreased) DEGs in hippocampus were seen in both 12- and 24-month old mice (Figure 5).

To validate the results achieved from the RNA-sequencing analysis, we randomly selected four DEGs, which were changed significantly (LFC $\geq 2.2$ or $\leq-2.2$ ), in both 12 - and 24 -month old mice, for quantitative RT-PCR assay. As expected, the amount of Igfbpl1 RNA in the hippocampus and the amount of Ostn RNA in the ACC were markedly reduced (Figures 6A,B), while the level of Klk6 RNA in the amygdala and the level of Defb1 RNA in the ACC were significantly increased in both 12- and 24-month old mice (Figures 6C,D).

\section{Functional Enrichment Analysis of the Differentially Expressed Genes in Aging Mice}

Database for Annotation, Visualization, and Integrated Discovery bioinformatics database was used to analyze the Gene Ontology analysis and categorize the DEGs based on the distinct processes. Figure 7 showed the analysis of DEGs for biological processes. The DEGs in the ACC from both 12and 24-month old mice were enriched highly in the immune response. The DEGs in the amygdala from 12-month old mice were enriched predominantly in immune system development, hemopoietic organ development, and hemopoiesis. The DEGs in both amygdala and hippocampus from 24-month old mice were enriched mainly in biological adhesion, cell adhesion, and immune response. The DEGs in the hippocampus from 12-month old mice were enriched largely in the regulation of cell proliferation, cell motion, cell motility, and localization of cell. We also categorized these DEGs according to their molecular functions (Supplementary Figure 2). The DEGs in ACC from 12 -month old mice and in the hippocampus from both 12and 24-month old mice were enriched mostly in calcium ion binding function. The DEGs in the amygdala from 24-month old mice were enriched predominantly in carbohydrate-binding function. The DEGs in the amygdala from 12-month old mice were enriched mainly in enzyme inhibitory activity, peptidase inhibitory activity, carbohydrate-binding, and endopeptidase inhibitory activity functions. The DEGs in ACC from 24-month old mice were enriched mainly in peptide binding, peptide receptor activity, cytokine activity, and hormone activity functions. Finally, we divided these DEGs according to their cellular components (Supplementary Figure 3). In both 12and 24-month old mice, the DEGs in ACC were enriched predominantly in the plasma membrane and extracellular region, the DEGs in the amygdala mainly in the extracellular region 

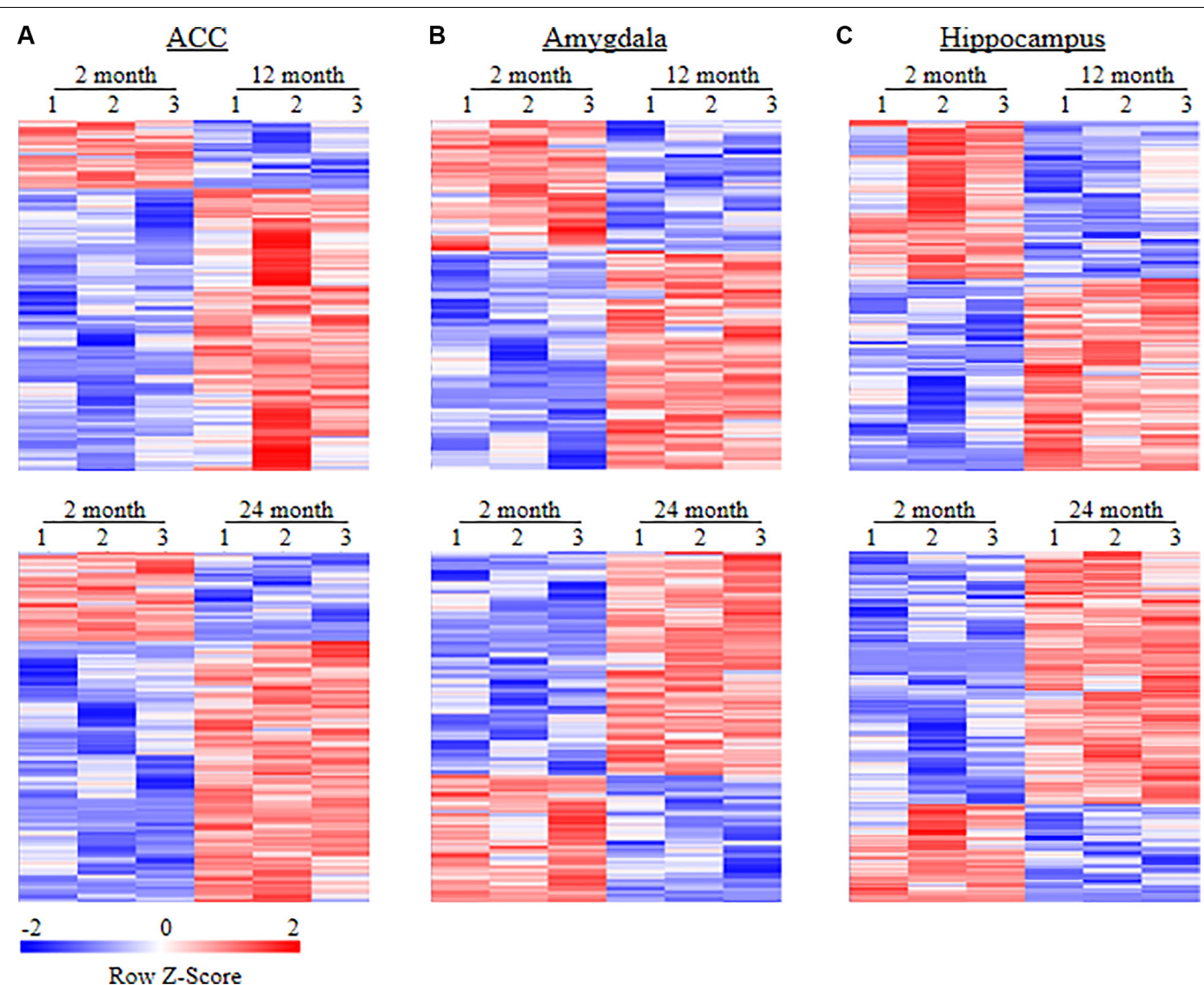

Row Z-Score

FIGURE 4 | Heatmaps of differentially expressed genes (DEGs) in the ACC (A), amygdala (B) and hippocampus (C) from 2-month old mice versus 12 -month old mice (top) or 24-month old mice (bottom). Colors in the heatmaps indicate the Row Z-score among the different data sets. High expression is shown by the red color spectrum, and low expression is shown by the blue. $N=3$ biological repeats ( 3 mice)/age.

and the DEGs in hippocampus mostly in intrinsic and integral to the membrane.

\section{Depression, Anxiety, and Cognitive Dysfunction-Associated DEGs in Aging Mice}

To further analyze the relationship between DEGs and behavioral changes with aging, we used the GeneCards database and CTD database to search cognitive dysfunction, depression, and anxiety-related genes. Approximately 2000 genes were selected according to the relevance score as data sets for the subsequent analysis. Given that ACC and amygdala play an important role in the genesis of depression and anxiety and that hippocampus is a key region associated with cognitive dysfunction $^{8-13}$, we compared the depression- and anxietyrelated gene data sets with the DEGs in the ACC and amygdala, and the cognitive dysfunction-related gene data set with the DEGs in the hippocampus. About 47 and 96 depression-related DEGs in the ACC and 25 and 103 depression-related DEGs in the amygdala were found in 12- and 24-month old mice, respectively (Supplementary Material 1). Approximately 205 and 124 anxiety-related DEGs in the ACC and 110 and 128 anxiety-related DEGs in the amygdala were seen in 12- and 24-month old mice, respectively (Supplementary Material 1). Finally, there were about 125 and 128 cognitive dysfunctionrelated DEGs in the hippocampus of 12- and 24-month old mice, respectively (Supplementary Material 1).

Given that the depression/anxiety-related behaviors and cognitive dysfunction were associated with inflammation, apoptosis, oxidative stress, synaptic plasticity, glutamate receptor pathway, and DNA methylation (Makhija and Karunakaran, 2013; Sheng and Erturk, 2014; Cai et al., 2015; Januar et al., 2015), we further analyzed the functions of the DEGs in the ACC, amygdala and hippocampus. All depression/anxiety/cognitive-related DEGs in three brain regions were associated with inflammation in both 12 - and 24-month old mice (Supplementary Table 2). In addition, among the depression-related DEGs in the ACC, about 96, 87, 26,66 , and $46 \%$ from 12 -month old mice and about 89,84 , 36,67 , and $92 \%$ from 24 -month old mice were associated with apoptosis, oxidative stress, synaptic plasticity, glutamate receptor pathway, and DNA methylation, respectively (Supplementary Table 2). Among the depression-related DEGs in the amygdala, approximately $92,80,36,72$, and $88 \%$ from 12 -month old mice and approximately $87,88,41,71$, and $91 \%$ from 24 -month old 

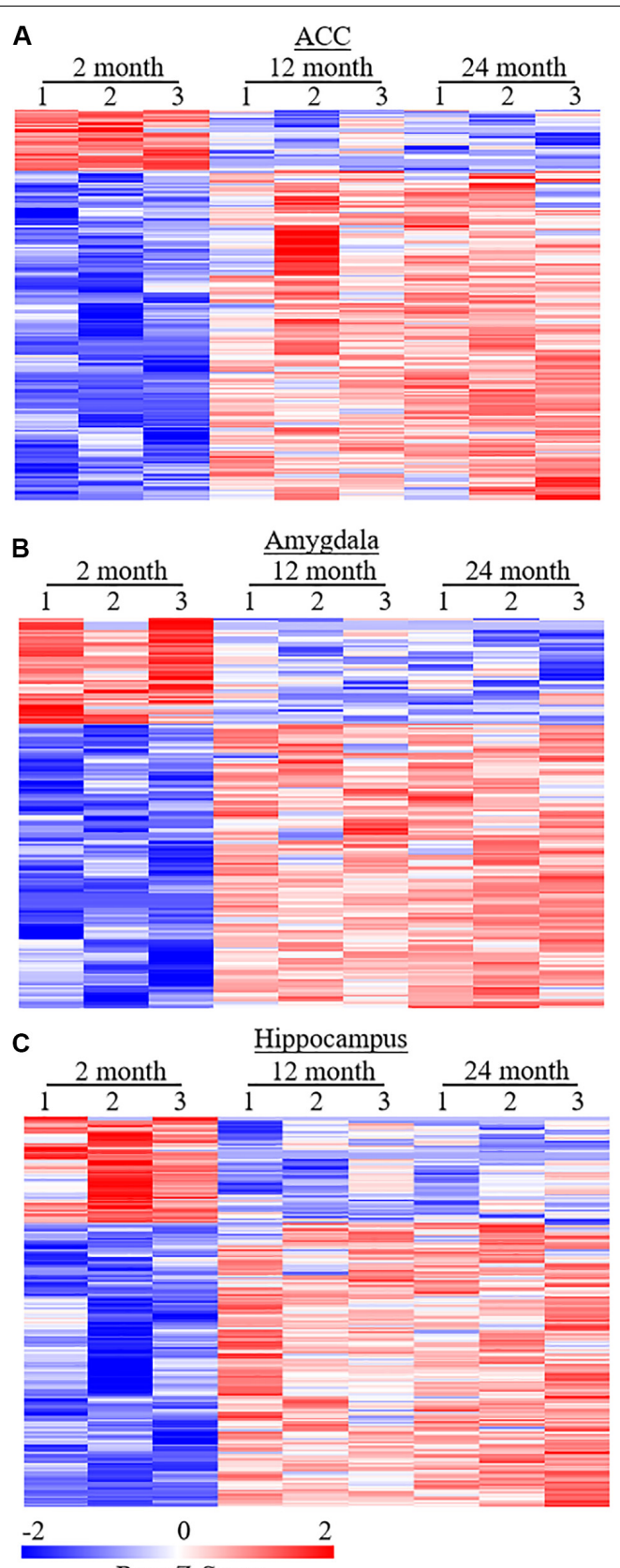

Row Z-Score

FIGURE $\mathbf{5}$ | Heatmaps of differentially expressed genes (DEGs) in the ACC (A), amygdala (B), and hippocampus (C) from both 12- and 24-month old mice versus 2 -month old mice. Colors in the heatmaps indicate the Row $Z$-score among the different data sets. High expression is shown by the red color spectrum, and low expression is shown by the blue. $N=3$ biological repeats (3 mice)/age.

mice were associated with apoptosis, oxidative stress, synaptic plasticity, glutamate receptor pathway, and DNA methylation, respectively (Supplementary Table 2). Among the anxietyrelated DEGs in AAC, about 44, 36, and 63\% from 12-month old mice and approximately 49, 42, and 60\% from 24 -month old mice were associated with oxidative stress, glutamate receptor
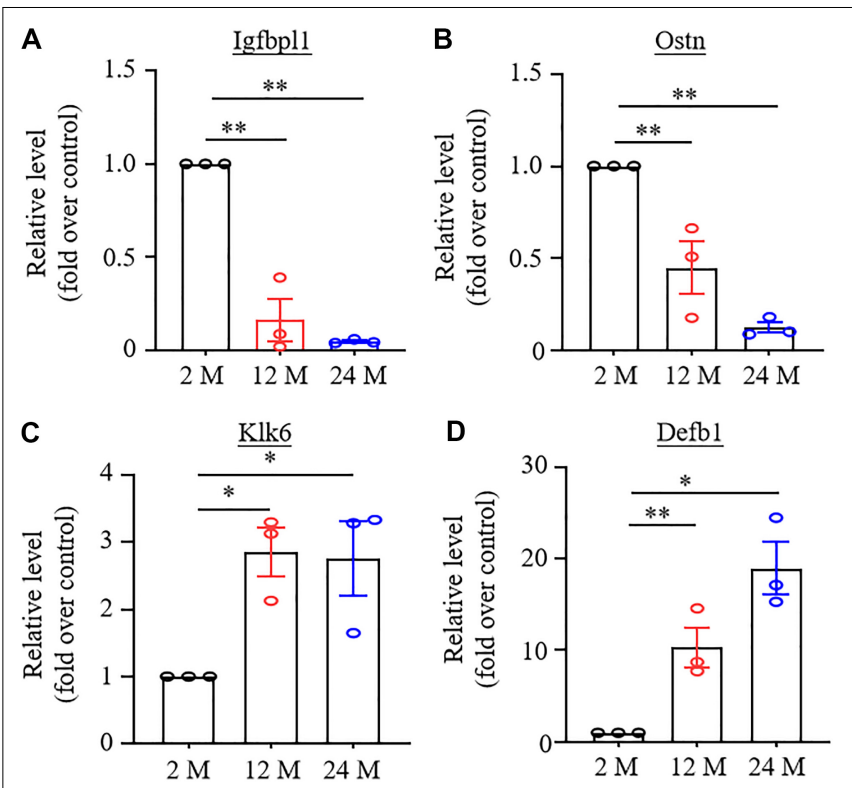

FIGURE 6 | Verification of the changes in expression of some differentially expressed genes using real-time reverse transcription polymerase chain reaction (RT-PCR) analysis. $N=3$ biological repeats (3 mice)/age. Data were shown as mean \pm SEM. (A,B) Expression of lgfbp/1 mRNA in the hippocampus (A) and Ostn mRNA in ACC (B) were significantly reduced in both 12- and 24-month old mice as compared to 2-month old mice.

${ }^{* *} P<0.01$ versus the corresponding 2 -month old mice by one-way ANOVA with repeated measures followed by post hoc Tukey test. (C,D) Expression of KIk6 mRNA in amygdala (C) and Defb 1 mRNA in ACC (D) were significantly increased in 12- and 24-month old mice as compared to 2-month old mice. ${ }^{*} P<0.05,{ }^{* *} P<0.01$ versus 2 -month old mice by one-way ANOVA with repeated measures followed by post hoc Tukey test.

pathway, and DNA methylation, respectively (Supplementary Table 2). Among the anxiety-related DEGs in amygdala, about 23,30 , and $56 \%$ from 12 -month old mice and approximately 64,46 , and $67 \%$ from 24 -month old mice were associated with oxidative stress, glutamate receptor, and DNA methylation, respectively (Supplementary Table 2 ). Among the cognitive dysfunction-related DEGs in the hippocampus, about 78, 66, 34, 53 , and $75 \%$ from 12 -month old mice and about 79, 70, 34, 61, and $73 \%$ from 24 -month old mice were associated with apoptosis, oxidative stress, synaptic plasticity, glutamate receptor pathway, and DNA methylation, respectively (Supplementary Table 2).

\section{Depression, Anxiety and Cognitive Dysfunction-Associated Genes With Differentially Expressed Isoforms in Aging Mice}

We also used the same strategy to analyze the relationship between the genes with differentially expressed isoforms and behavioral changes with aging. About 525 and 507 depressionrelated genes in the ACC and 448 and 564 depression-related genes in the amygdala were found in 12- and 24-month old mice, respectively (Supplementary Material 2). Approximately 1918 and 1998 anxiety-related genes in the ACC and 1706 and 


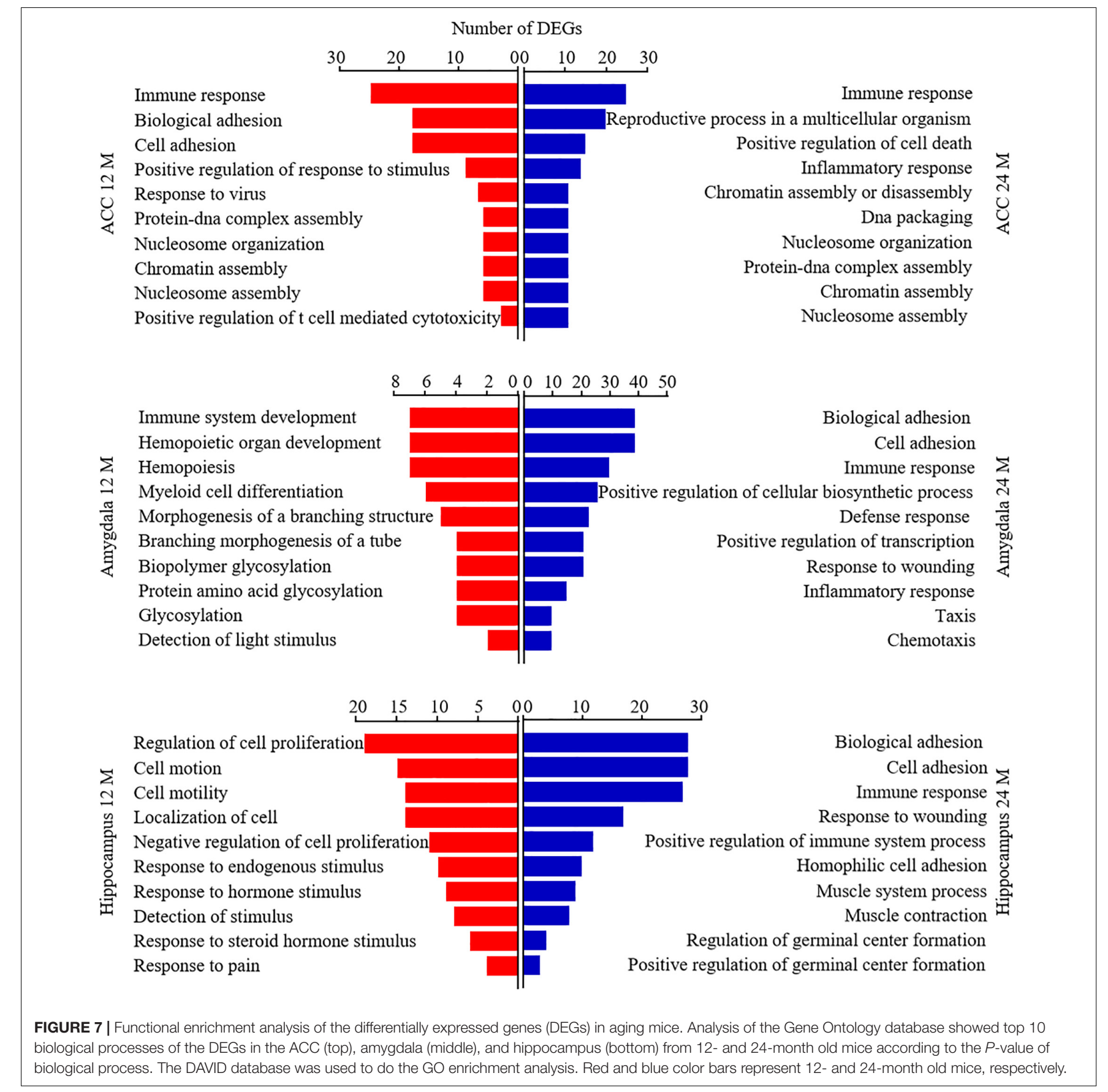

1991 anxiety-related genes in the amygdala were seen in 12- and 24-month old mice, respectively (Supplementary Material 2). Finally, there were about 920 and 881 cognitive dysfunctionrelated genes in the hippocampus from 12- and 24-month old mice, respectively (Supplementary Material 2).

Further analysis revealed that all of these depression/anxiety/cognitive-related genes with differentially expressed isoforms in these three regions were associated with inflammation in both 12 - and 24-month old mice (Supplementary Table 3). In addition, among the depressionrelated genes with differentially expressed isoforms in ACC, about $89,80,42,67$, and $91 \%$ from 12 -month old mice and about $87,80,38,64$, and $87 \%$ from 24 -month old mice were associated with apoptosis, oxidative stress, synaptic plasticity, glutamate receptor pathway, and DNA methylation, respectively (Supplementary Table 3). Among the depression-related genes with differentially expressed isoforms in amygdala, approximately $89,80,40,65$, and $88 \%$ from 12 -month old mice and approximately $87,78,41,65$, and $89 \%$ from 24 -month old mice were associated with apoptosis, oxidative stress, synaptic plasticity pathway, glutamate receptor pathway, and DNA methylation, respectively (Supplementary Table 3). Among 
the anxiety-related genes with differentially expressed isoforms in AAC, about 56, 39, and $71 \%$ from 12 -month old mice and approximately 55,38 , and $67 \%$ from 24 -month old mice were associated with oxidative stress, glutamate receptor pathway, and DNA methylation, respectively (Supplementary Table 3). Among the anxiety-related genes with differentially expressed isoforms in amygdala, about 58, 38, and 69\% from 12-month old mice and approximately 57, 39, and 70\% from 24 -month old mice were associated with oxidative stress, glutamate receptor pathway, and DNA methylation, respectively (Supplementary Table 3). Among the cognitive dysfunction-related genes with differentially expressed isoforms in hippocampus, about 81, 71, 35,55 , and $80 \%$ from 12 -month old mice and about $82,74,33,54$, and $80 \%$ from 24 -month old mice were associated with apoptosis, oxidative stress, synaptic plasticity, glutamate receptor pathway, and DNA methylation, respectively (Supplementary Table 3).

\section{Establishing a PPI Network to Analyze Protein-Protein Interactions}

To explore the relationship between the DEGs and aging pathology, we performed the PPI network analysis using the STRING database (Figure 8). Due to the involvement of a large number of DEGs in the network, the top 50 DEGs from each region were selected to draw the PPI network according to the connection degree. A serial of networks was generated, and the hub genes were shown in the network. Cxcl10, an important chemokine, was the central node of the network in the ACC of 12- and 24-month old mice (Figures 8A,B). Serpinala, Serpinf2, Igf2, and Pomc played a crucial role in the network of the amygdala in 12-month old mice (Figure 8C). In contrast, Serpin family and Serpinb1b were central molecules in the network of the amygdala in 24-month old mice (Figure 8D). Moreover, there were 10 genes (Gcgr, Shh, Fgg, Plg, Fgf2, Cxcr4, Cxcl10, Ftcd, Edn1, Itgax, and $\mathrm{Cxcl5}$ ) played a critical role in the network of the hippocampus of 12- and 24-month old mice (Figures 8E,F). The network also showed that the inflammationand apoptosis-related genes occupied a vital position in the PPI networks (Figure 8).

\section{Association of Several DEGs With Depression-, Anxiety-, or Cognitive Dysfunction-Related Behaviors in Aging Mice}

Finally, we carried out the Pearson method to analyze the correlation between mRNA levels of three selected DEGs from the ACC/amygdala and the depression/anxiety-like behaviors or between mRNA levels of three selected DEGs from hippocampus and cognitive dysfunction. In the ACC, the levels of Esr1, Crhr2, and Tgif1 mRNAs were positively associated with the immobile time of the forced swimming and TSTs $(r=0.848 \sim 0.977$, $P=0.033 \sim 0.001 ;$ Supplementary Table 4$)$. The amount of Tshr mRNA was negatively associated with the percentage of distance in central $(r=-0.939, P=0.006)$ and the entries in central $(r=-0.931, P=0.007)$ in OFT as well as the percentage of entries in open arms $(r=-0.968, P=0.001)$ and the percentage of time in open arms $(r=-0.833, P=0.039)$ in EPM test (Supplementary Table 4). The level of Esr1 mRNA was negatively associated with the percentages of distance in central $(r=-0.856$ and $P=0.030)$ and the entries in central $(r=-0.899, P=0.015)$ in OFT and with the percentages of entries in open arms in EPM test $(r=-0.909, P=0.012)$. Interestingly, the amount of Crhr2 mRNA was only negatively related to the entries in central in OFT $(r=-0.876, P=0.022)$ (Supplementary Table 4). In the amygdala, the immobile time of the tail suspension and FSTs was positively associated with the level of $V d r$ mRNA $(r=0.886, P=0.019 ; r=0.872, P=0.023)$ and negatively related to the amount of Kif1l mRNA $(r=-0.850$, $P=0.032 ; r=-0.823, P=0.044$ ) (Supplementary Table 4). Unexpectedly, no significant associations were observed between Pomc mRNA expression and these depression-like behaviors in mice (Supplementary Table 4). In addition, the levels of Bcl2l10, Serpinf2, and Piwil4 mRNAs displayed high positive correlations to the parameters indicated above in the OFT $(r=-0.858 \sim$ -0.884 and $0.828 \sim 0.944, P=0.042 \sim 0.005$; Supplementary Table 4). In the EPM test, the level of Serpinf2 mRNA was positively relevant to the percentage of entries $(r=0.852$, $P=0.031)$ and the percentage of time $(r=0.860, P=0.028)$ in open arms, the amount of Bcl2l10 was negatively associated with the percentage of entries in open arms $(r=-0.852, P=0.031)$ and the level of Piwil4 mRNA was unrelated to either parameter (Supplementary Table 4). In the hippocampus, the level of $\mathrm{Plg}$ mRNA was negatively associated with the percentages of spontaneous alternation in the Y-maze spontaneous alternation tests $(r=-0.892, P=0.017)$, recognition in exploring new objects $3 \mathrm{~h}(r=-0.956, P=0.003)$ and $24 \mathrm{~h}(r=-0.854, P=0.030)$ after training in the NOR test, and the numbers of platform crossing in the MWM tests $(r=-0.889, P=0.018)$ (Supplementary Table 5). In contrast, the level of Dbh mRNA was positively related to the percentages of spontaneous alternation in the Y-maze spontaneous alternation tests $(r=0.884, P=0.019)$ and recognition in exploring new objects $3 \mathrm{~h}$ after training in the NOR test ( $r=0.855, P=0.030$ ) (Supplementary Table 5). The level of Prph mRNA was negatively associated only with the numbers of platform crossing $1 \mathrm{~h}$ after training in the MWM test $(r=-0.869$, $P=0.025)$ (Supplementary Table 5).

\section{DISCUSSION}

Despite intensive research into the molecular and biological mechanisms of aging and aging-related diseases during past decades, aging and aging-related diseases are poorly managed by current drugs. In the present study, we used a mouse model of natural aging to mimic the different aging stages in humans to search the aging symptoms-associated genes in ACC, amygdala and hippocampus. Behavior tests were first carried out to verify depression/anxiety-like behaviors and learning, memory and cognitive impairments in the 12- and 24-month old mice. Bioinformatical analyses further showed that all DEGs and the genes with differentially expressed isoforms identified in ACC, hippocampus and amygdala of two groups of aging mice were closely related to the neuro-inflammation. Our findings demonstrate that cognitive impairment and emotional 


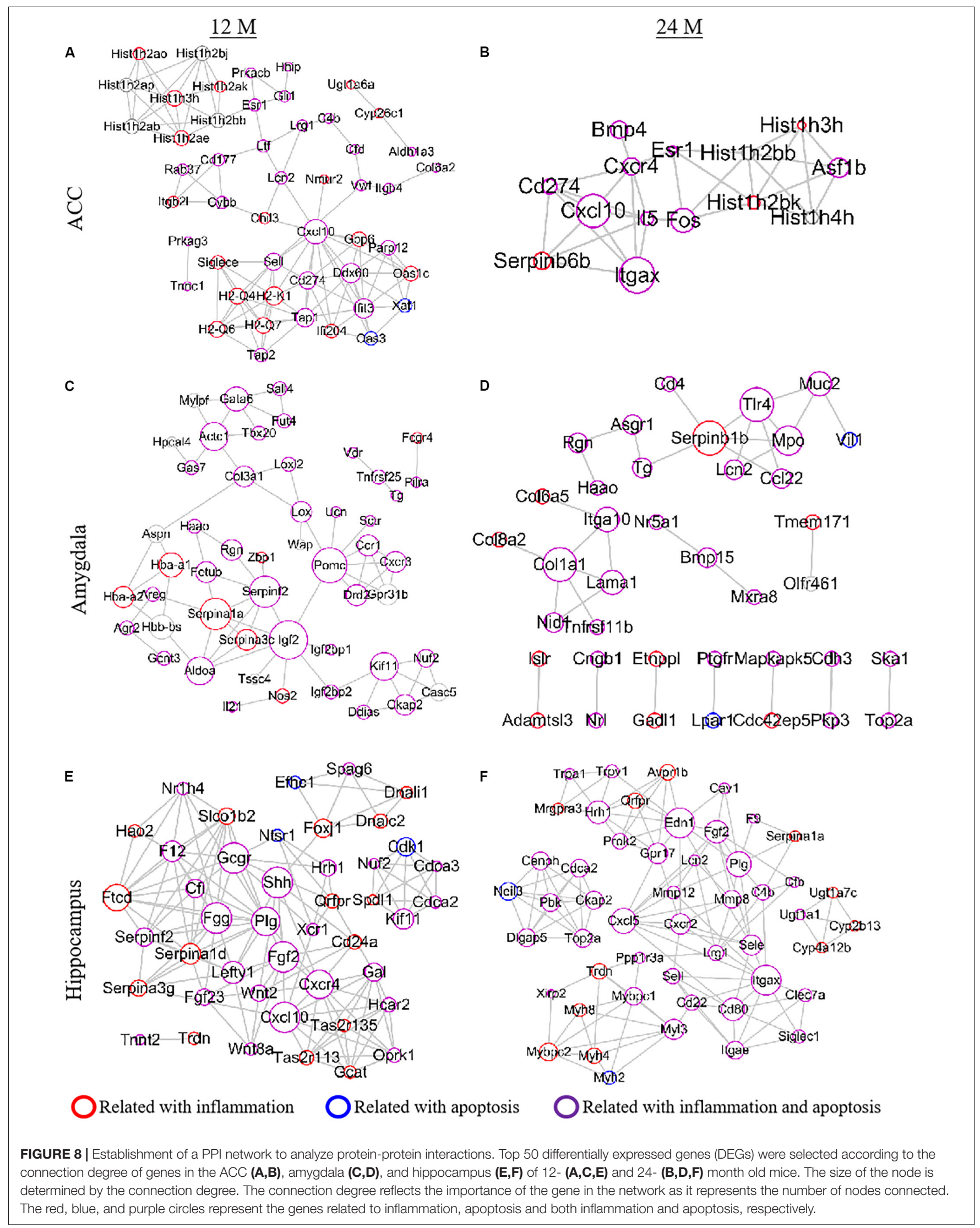


disorders with aging are likely corresponding to the changes in neuroinflammation-related genes.

In the present study, we found that the mice of 12- and 24month old showed the progressive loss in episodic-like memory with aging demonstrated by NOR assay. This observation was consistent with the previous reports that the object recognition memory seemed to be impaired as early as the age of 12 months (Belblidia et al., 2018). Moreover, in both groups of aged mice, spatial memory with aging was impaired evidenced by the decreased percentage of spontaneous alternation during the Y-maze test and by the prolonged escape latency, as well as the reduced numbers of crossing the position where the platform located in the training sessions during MWM test. Consistent with a previous study (Bach et al., 1999), 24-month old mice exhibited a decline in locomotor activity evaluated by the decrease in total distance traveled in OFT assay and reduced number of the entry in EPM test.

Although the mechanism of age-related emotional dysfunction remains controversial in many studies (Schulz et al., 2007; Malatynska et al., 2012), increasing evidence suggests that cognitive impairment in the aged population often accompanied by depression and anxiety disorders (Kuring et al., 2018) 52. Our findings demonstrated that the immobility time was increased in both forced swimming and TSTs in aging mice. However, in the OFT, there were no apparent changes in exploration activity between 2- and 12-month old mice, indicating depression-like behaviors mainly affected the later stage of the aging process. Anxiety-like behavior in aged mice was demonstrated by the decreased percentages of open arm entries and the time spent in open arms using EPM test, an observation in agreement with the clinical studies that memory loss and dementia were often accompanied by neuropsychiatric symptoms in abnormal aging patients (Peprah and McCormack, 2019).

The previous studies on age-related changes in pain sensitivity response are controversial (Yezierski, 2012). Several reports showed that pain perception is diminished in older people (Gibson and Helme, 2001; Gibson and Farrell, 2004), whereas other observations revealed the pain threshold increased in old age (Lautenbacher, 2012). Comprehensive reviews by EI Tumi et al. recently suggest that that old adult may be more sensitive to mechanically evoked pain but not heat-evoked pain than young adults (El Tumi et al., 2017). The present study showed no significant changes in basal sensitivities in response to evoked mechanical and thermal stimuli in both 12- and 24-month old mice compared to 2-month old mice, which is in line with the previous reports in the animals (Fahlstrom et al., 2012; Shoji et al., 2016; Millecamps et al., 2019; Shoji and Miyakawa, 2019). The reasons of why preclinical studies are inconsistent with clinical observations are unclear, but might be due to the inconsistent stages of the aging process between humans and animals as well as the limited measurement methods, parameters, and dimensions in animal studies.

Despite more and more attention focused on aging and agerelated disorders in clinical and preclinical investigations, the underlying mechanisms remain unclear. To gain insight into the aging process and provide the potential targets, gene expression profile changes from aging mice were detected in the present study. We identified about 634 and 1078 DEGs in ACC, 453 and 1015 DEGs in the amygdala, 884 and 1054 DEGs in the hippocampus in the 12- and 24-month old mice, respectively. We also identified many genes with differentially expressed isoforms in these three brain regions in the 12- and 24-month old mice. Further function analysis of DEGs and the genes with differentially expressed isoforms revealed that many of them in the ACC and amygdala were mapped to depression- and anxietyrelated genes, respectively and that a lot of them in hippocampus were mapped to cognitive dysfunction-related genes from both 12- and 24-month old mice. All of these mapped DEGs and the genes with differentially expressed isoforms were closely related to neuroinflammation. Some of them were associated with apoptosis, oxidative stress, synaptic plasticity, glutamate receptor pathway or DNA methylation. We found that Cnr2 and Tlr4 genes related to neuroinflammation were robustly upregulated in the ACC and amygdala of aging mice, suggesting that these two genes play an important role in the genesis of depression and anxiety during aging. This conclusion is strongly supported by the previous studies that showed that knockout of Cnr2 in the brain could attenuate psychomotor, depression- and anxietylike behaviors in mice (Liu Q.R. et al., 2017) and that blocking Tlr4 could reduce the anxiety- and depression-like behaviors (Strekalova et al., 2015; Zhang et al., 2020). In addition, we also found that the other two neuroinflammation-related genes Fgf2 and Cbln 1 were differentially expressed in the hippocampus of aging mice, both of which have been demonstrated to affect fear conditioning and spatial memory processes in aged animals (Hirai et al., 2005).

It should be pointed out that present study has several limitations to be considered. Firstly, although we reported significant correlations of several selected DEGs in AAC, amygdala and hippocampus with depression-, anxiety-, or cognitive dysfunction-like behaviors, these correlations do not imply causation. Thus, we should be very careful for the interpretation of our data and perform more stringent researches to examine the roles of these candidate genes in depression, anxiety or cognitive dysfunction. Secondly, the expressional levels of some selected DEGs were not significantly associated with some parameters in behavioral tests or had no correlations with depression-, anxiety-, or cognitive dysfunction-like behaviors. These non-associations/non-correlations may be attributed to low number of the samples per group ( $n=3$ repeats/group). Increasing number of the samples per group may improve correlation efficient. Thirdly, our research on depression, anxiety and cognitive dysfunction in aging focused on male mice. There have been few studies on female mice so far. However, gender-specific effect of DEGs on depression, anxiety and cognitive dysfunction in aging could exist and remains to be addressed in the future.

\section{CONCLUSION}

In conclusion, we demonstrated that progressive memory impairment and emotional disorders with aging were relevant 
to the neuroinflammation-related DEGs and the genes with differentially expressed isoforms in ACC, amygdala and hippocampus. Our findings may uncover a new layer of agingrelated transcriptional regulation and be helpful for the drug development for management of aging and age-related disorders.

\section{DATA AVAILABILITY STATEMENT}

All sequencing data are available in NCBI database (accession number: SRP271007).

\section{ETHICS STATEMENT}

The animal study was reviewed and approved by the Animal Care and Use Committee of Zhengzhou University.

\section{AUTHOR CONTRIBUTIONS}

YA and Y-XT conceived the project, supervised all experiments, and edited the draft. ML, SS, JC, XM, WZ, JY, Y-XT, and YA assisted with experimental design. ML, SS, and XM took care of feeding and maintenance of old mice. ML, SS, and WC carried

\section{REFERENCES}

Alam, A., Hana, Z., Jin, Z., Suen, K. C., and Ma, D. (2018). Surgery, neuroinflammation and cognitive impairment. Ebiomedicine 37, 547-556. doi: 10.1016/j.ebiom.2018.10.021

Bach, M. E., Barad, M., Son, H., Zhuo, M., Lu, Y. F., Shih, R., et al. (1999). Agerelated defects in spatial memory are correlated with defects in the late phase of hippocampal long-term potentiation in vitro and are attenuated by drugs that enhance the cAMP signaling pathway. Proc. Natl. Acad. Sci. U.S.A. 96, 5280-5285. doi: 10.1073/pnas.96.9.5280

Beard, J. R., Officer, A., de Carvalho, I. A., Sadana, R., Pot, A. M., Michel, J. P., et al. (2016). The World report on ageing and health: a policy framework for healthy ageing. Lancet 387, 2145-2154. doi: 10.1016/S0140-6736(15)00516-4

Belblidia, H., Leger, M., Abdelmalek, A., Quiedeville, A., Calocer, F., Boulouard, M., et al. (2018). Characterizing age-related decline of recognition memory and brain activation profile in mice. Exp. Gerontol. 106, 222-231. doi: 10.1016/j. exger.2018.03.006

Cai, S., Huang, S., and Hao, W. (2015). New hypothesis and treatment targets of depression: an integrated view of key findings. Neurosci. Bull. 31, 61-74. doi: 10.1007/s12264-014-1486-4

Chao, O. Y., Huston, J. P., Li, J. S., Wang, A. L., and de Souza Silva, M. A. (2016). The medial prefrontal cortex-lateral entorhinal cortex circuit is essential for episodic-like memory and associative object-recognition. Hippocampus 26, 633-645. doi: 10.1002/hipo.22547

Costa-Ferreira, W., Morais-Silva, G., Gomes-de-Souza, L., Marin, M. T., and Crestani, C. C. (2019). The AT1 receptor antagonist losartan does not affect depressive-like state and memory impairment evoked by chronic stressors in rats. Front. Pharmacol. 10:705. doi: 10.3389/fphar.2019. 00705

Cribbs, D. H., Berchtold, N. C., Perreau, V., Coleman, P. D., Rogers, J., Tenner, A. J., et al. (2012). Extensive innate immune gene activation accompanies brain aging, increasing vulnerability to cognitive decline and neurodegeneration: a microarray study. J. Neuroinflamm. 9:179. doi: 10.1186/1742-2094-9-179

Davalli, P., Mitic, T., Caporali, A., Lauriola, A., and D'Arca, D. (2016). ROS, cell senescence, and novel molecular mechanisms in aging and agerelated diseases. Oxid. Med. Cell Longev. 2016:3565127. doi: 10.1155/2016/356 5127 out behavioral tests. ML performed tissue collection and RT-PCR assay. ML, SG, and YX analyzed the data and wrote the draft of manuscript. All authors read and discussed the manuscript.

\section{FUNDING}

This work was supported by the start-up fund from Zhengzhou University to YA, National Natural Science Foundation of China 81870864 to XM, and National Natural Science Foundation of China 81671091 to JC.

\section{ACKNOWLEDGMENTS}

The authors thank Dr. Xiang Gao and Mrs. Tingting Wang for their supports in RNA sequencing.

\section{SUPPLEMENTARY MATERIAL}

The Supplementary Material for this article can be found online at: https://www.frontiersin.org/articles/10.3389/fcell.2020.00814/ full\#supplementary-material

El Tumi, H., Johnson, M. I., Dantas, P. B. F., Maynard, M. J., and Tashani, O. A. (2017). Age-related changes in pain sensitivity in healthy humans: A systematic review with meta-analysis. Eur. J. Pain 21, 955-964. doi: 10.1002/ejp.1011

Etkin, A., Egner, T., Peraza, D. M., Kandel, E. R., and Hirsch, J. (2006). Resolving emotional conflict: a role for the rostral anterior cingulate cortex in modulating activity in the amygdala. Neuron 51, 871-882. doi: 10.1016/j.neuron.2006. 07.029

Ewbank, M. P., Passamonti, L., Hagan, C. C., Goodyer, I. M., Calder, A. J., and Fairchild, G. (2018). Psychopathic traits influence amygdala-anterior cingulate cortex connectivity during facial emotion processing. Soc. Cogn. Affect. Neurosci. 13, 525-534. doi: 10.1093/scan/nsy019

Fahlstrom, A., Zeberg, H., and Ulfhake, B. (2012). Changes in behaviors of male C57BL/6J mice across adult life span and effects of dietary restriction. Age 34, 1435-1452. doi: 10.1007/s11357-011-9320-7

Fougere, B., Boulanger, E., Nourhashemi, F., Guyonnet, S., and Cesari, M. (2017). Chronic inflammation: accelerator of biological aging. J. Gerontol. A Biol. Sci. Med. Sci. 72, 1218-1225. doi: 10.1093/gerona/glw240

Franco, R., Martinez-Pinilla, E., Navarro, G., and Zamarbide, M. (2017) Potential of GPCRs to modulate MAPK and mTOR pathways in Alzheimer's disease. Prog. Neurobiol. 149-150, 21-38. doi: 10.1016/j.pneurobio.2017. 01.004

Frisoni, G. B., Rozzini, L., Gozzetti, A., Binetti, G., Zanetti, O., Bianchetti, A., et al. (1999). Behavioral syndromes in Alzheimer's disease: description and correlates. Dement. Geriatr. Cogn. Disord. 10, 130-138. doi: 10.1159/00001 7113

Gibson, S. J., and Farrell, M. (2004). A review of age differences in the neurophysiology of nociception and the perceptual experience of pain. Clin. J. Pain 20, 227-239. doi: 10.1097/00002508-200407000-00004

Gibson, S. J., and Helme, R. D. (2001). Age-related differences in pain perception and report. Clin. Geriatr. Med. 17, 433-456. doi: 10.1016/s0749-0690(05)70 079-3

Gurkar, A. U., and Niedernhofer, L. J. (2015). Comparison of mice with accelerated aging caused by distinct mechanisms. Exp. Gerontol. 68, 43-50. doi: 10.1016/j. exger.2015.01.045

Harada, C. N., Natelson Love, M. C., and Triebel, K. L. (2013). Normal cognitive aging. Clin. Geriatr. Med. 29, 737-752. doi: 10.1016/j.cger.2013. 07.002 
Hekmatimoghaddam, S., Dehghani Firoozabadi, A., Zare-Khormizi, M. R., and Pourrajab, F. (2017). Sirtl and Parpl as epigenome safeguards and microRNAs as SASP-associated signals, in cellular senescence and aging. Ageing Res. Rev. 40, 120-141. doi: 10.1016/j.arr.2017.10.001

Herrup, K. (2010). Reimagining Alzheimer's disease-an age-based hypothesis. J. Neurosci. 30, 16755-16762. doi: 10.1523/JNEUROSCI.4521-10.2010

Hirai, H., Pang, Z., Bao, D., Miyazaki, T., Li, L., Miura, E., et al. (2005). Cbln1 is essential for synaptic integrity and plasticity in the cerebellum. Nat. Neurosci. 8, 1534-1541. doi: 10.1038/nn1576

Holmes, S. E., Hinz, R., Conen, S., Gregory, C. J., Matthews, J. C., AntonRodriguez, J. M., et al. (2018). Elevated translocator protein in anterior cingulate in major depression and a role for inflammation in suicidal thinking: a positron emission tomography study. Biol. Psychiatry 83, 61-69. doi: 10.1016/j.biopsych.2017.08.005

Hrdlickova, R., Toloue, M., and Tian, B. (2017). RNA-Seq methods for transcriptome analysis. Wiley Interdiscip. Rev. RNA 8:1364. doi: 10.1002/wrna. 1364

Inestrosa, N. C., and Varela-Nallar, L. (2014). Wnt signaling in the nervous system and in Alzheimer's disease. J. Mol. Cell Biol. 6, 64-74. doi: 10.1093/jmcb/mjt051

Januar, V., Saffery, R., and Ryan, J. (2015). Epigenetics and depressive disorders: a review of current progress and future directions. Int. J. Epidemiol. 44, 13641387. doi: 10.1093/ije/dyu273

Kanasi, E., Ayilavarapu, S., and Jones, J. (2016). The aging population: demographics and the biology of aging. Periodontology 72, 13-18. doi: 10.1111/ prd. 12126

Killgore, W. D., and Yurgelun-Todd, D. A. (2004). Activation of the amygdala and anterior cingulate during nonconscious processing of sad versus happy faces. Neuroimage 21, 1215-1223. doi: 10.1016/j.neuroimage.2003.12.033

Klinger, K., Gomes, F. V., Rincon-Cortes, M., and Grace, A. A. (2019). Female rats are resistant to the long-lasting neurobehavioral changes induced by adolescent stress exposure. Eur. Neuropsychopharmacol. 29:134. doi: 10.1016/j.euroneuro. 2019.07.134

Kuring, J. K., Mathias, J. L., and Ward, L. (2018). Prevalence of depression, anxiety and PTSD in people with dementia: a systematic review and meta-analysis. Neuropsychol. Rev. 28, 393-416. doi: 10.1007/s11065-018-9396-2

Lautenbacher, S. (2012). Experimental approaches in the study of pain in the elderly. Pain Med. 13(Suppl. 2), S44-S50. doi: 10.1111/j.15264637.2012.01326.x

Liu, E., Xie, A. J., Zhou, Q., Li, M., Zhang, S., Li, S., et al. (2017). GSK-3beta deletion in dentate gyrus excitatory neuron impairs synaptic plasticity and memory. Sci. Rep. 7:5781. doi: 10.1038/s41598-017-06173-4

Liu, Q. R., Canseco-Alba, A., Zhang, H. Y., Tagliaferro, P., Chung, M., Dennis, E., et al. (2017). Cannabinoid type 2 receptors in dopamine neurons inhibits psychomotor behaviors, alters anxiety, depression and alcohol preference. Sci. Rep. 7:17410. doi: 10.1038/s41598-017-17796-y

Liu, W., Ge, T., Leng, Y., Pan, Z., Fan, J., Yang, W., et al. (2017). The role of neural plasticity in depression: from hippocampus to prefrontal cortex. Neural Plast. 2017:6871089. doi: 10.1155/2017/6871089

Liu, K., Fu, Q., Liu, Y., and Wang, C. (2019). An integrative bioinformatics analysis of microarray data for identifying hub genes as diagnostic biomarkers of preeclampsia. Biosci. Rep. 39:187. doi: 10.1042/BSR20190187

Lueptow, L. M. (2017). Novel object recognition test for the investigation of learning and memory in mice. J. Vis. Exp. 2017:55718. doi: 10.3791/55718

Makhija, K., and Karunakaran, S. (2013). The role of inflammatory cytokines on the aetiopathogenesis of depression. Aust. N. Z. J. Psychiatry 47, 828-839. doi: $10.1177 / 0004867413488220$

Malatynska, E., Steinbusch, H. W., Redkozubova, O., Bolkunov, A., Kubatiev, A., Yeritsyan, N. B., et al. (2012). Anhedonic-like traits and lack of affective deficits in 18-month-old C57BL/6 mice: implications for modeling elderly depression. Exp. Gerontol. 47, 552-564. doi: 10.1016/j.exger.2012.04.010

Martin, D. M., McClintock, S. M., Forster, J. J., Lo, T. Y., and Loo, C. K. (2017). Cognitive enhancing effects of rTMS administered to the prefrontal cortex in patients with depression: a systematic review and meta-analysis of individual task effects. Depress. Anxiety 34, 1029-1039. doi: 10.1002/da.2 2658

Mehta, N. D., Haroon, E., Xu, X., Woolwine, B. J., Li, Z., and Felger, J. C. (2018). Inflammation negatively correlates with amygdala-ventromedial prefrontal functional connectivity in association with anxiety in patients with depression: preliminary results. Brain Behav. Immun. 73, 725-730. doi: 10.1016/j.bbi.2018. 07.026

Millecamps, M., Shi, X. Q., Piltonen, M., Echeverry, S., Diatchenko, L., Zhang, J., et al. (2019). The geriatric pain experience in mice: intact cutaneous thresholds but altered responses to tonic and chronic pain. Neurobiol. Aging 89, 1-11. doi: 10.1016/j.neurobiolaging.2019.12.018

Peprah, K., and McCormack, S. (2019). Medical Cannabis for the Treatment of Dementia: A Review of Clinical Effectiveness and Guidelines. Ottawa, ON: Canadian Agency for Drugs and Technologies in Health.

Piel, J. H., Lett, T. A., Wackerhagen, C., Plichta, M. M., Mohnke, S., Grimm, O., et al. (2018). The effect of 5-HTTLPR and a serotonergic multi-marker score on amygdala, prefrontal and anterior cingulate cortex reactivity and habituation in a large, healthy fMRI cohort. Eur. Neuropsychopharmacol. 28, 415-427. doi: 10.1016/j.euroneuro.2017.12.014

Porsolt, R. D., Bertin, A., and Jalfre, M. (1978). "Behavioural despair" in rats and mice: strain differences and the effects of imipramine. Eur. J. Pharmacol. 51, 291-294. doi: 10.1016/0014-2999(78)90414-4

Reichwald, J., Danner, S., Wiederhold, K. H., and Staufenbiel, M. (2009). Expression of complement system components during aging and amyloid deposition in APP transgenic mice. J. Neuroinflamm. 6:35. doi: 10.1186/17422094-6-35

Rice, J., Coutellier, L., Weiner, J. L., and Gu, C. (2019). Region-specific interneuron demyelination and heightened anxiety-like behavior induced by adolescent binge alcohol treatment. Acta Neuropathol. Commun. 7:173. doi: 10.1186/ s40478-019-0829-9

Salthouse, T. (2012). Consequences of age-related cognitive declines. Annu. Rev. Psychol. 63, 201-226. doi: 10.1146/annurev-psych-120710-10 0328

Scalici, F., Caltagirone, C., and Carlesimo, G. A. (2017). The contribution of different prefrontal cortex regions to recollection and familiarity: a review of fMRI data. Neurosci. Biobehav. Rev. 83, 240-251. doi: 10.1016/j.neubiorev.2017. 10.017

Schulz, D., Huston, J. P., Buddenberg, T., and Topic, B. (2007). "Despair" induced by extinction trials in the water maze: relationship with measures of anxiety in aged and adult rats. Neurobiol. Learn. Mem. 87, 309-323. doi: 10.1016/j.nlm. 2006.09.006

Sheng, M., and Erturk, A. (2014). Long-term depression: a cell biological view. Philos. Trans. R. Soc. Lond. B Biol. Sci. 369:20130138. doi: 10.1098/rstb.2013. 0138

Shoji, H., and Miyakawa, T. (2019). Age-related behavioral changes from young to old age in male mice of a $\mathrm{C} 57 \mathrm{BL} / 6 \mathrm{~J}$ strain maintained under a genetic stability program. Neuropsychopharmacol. Rep. 39, 100-118. doi: 10.1002/npr2. 12052

Shoji, H., Takao, K., Hattori, S., and Miyakawa, T. (2016). Age-related changes in behavior in C57BL/6J mice from young adulthood to middle age. Mol. Brain 9:11. doi: 10.1186/s13041-016-0191-9

Strekalova, T., Evans, M., Costa-Nunes, J., Bachurin, S., Yeritsyan, N., Couch, Y., et al. (2015). Tlr4 upregulation in the brain accompanies depression- and anxiety-like behaviors induced by a high-cholesterol diet. Brain Behav. Immun. 48, 42-47. doi: 10.1016/j.bbi.2015.02.015

Sun, N., Youle, R. J., and Finkel, T. (2016). The mitochondrial basis of aging. Mol. Cell. 61, 654-666. doi: 10.1016/j.molcel.2016.01.028

Vorhees, C. V., and Williams, M. T. (2006). Morris water maze: procedures for assessing spatial and related forms of learning and memory. Nat. Protoc. 1, 848-858. doi: 10.1038/nprot.2006.116

Wadhwa, M., Chauhan, G., Roy, K., Sahu, S., Deep, S., Jain, V., et al. (2018). Caffeine and modafinil ameliorate the neuroinflammation and anxious behavior in rats during sleep deprivation by inhibiting the microglia activation. Front. Cell Neurosci. 12:49. doi: 10.3389/fncel.2018. 00049

Wen, J., Yang, Y., Wu, S., Wei, G., Jia, S., Hannaford, S., et al. (2020). Long noncoding RNA H19 in the injured dorsal root ganglion contributes to peripheral nerve injury-induced pain hypersensitivity. Transl. Perioper. Pain Med. 7, 176-184.

Xu, Y., Cui, S.-Y., Ma, Q., Shi, J., Yu, Y., Li, J.-X., et al. (2018). transresveratrol ameliorates stress-induced irritable bowel syndrome-like behaviors by regulation of brain-gut axis. Front. Pharmacol. 9:631. doi: 10.3389/fphar. 2018.00631 
Yan, W., Liu, J. F., Han, Y., Zhang, W., Luo, Y. X., Xue, Y. X., et al. (2018). Protein kinase Mzeta in medial prefrontal cortex mediates depressive-like behavior and antidepressant response. Mol. Psychiatry 23, 1878-1891. doi: 10.1038/mp.201 7.219

Yezierski, R. P. (2012). The effects of age on pain sensitivity: preclinical studies. Pain Med. 13, S27-S36. doi: 10.1111/j.1526-4637.2011. 01311.x

Zhang, K., Lin, W., Zhang, J., Zhao, Y., Wang, X., and Zhao, M. (2020). Effect of Toll-like receptor 4 on depressive-like behaviors induced by chronic social defeat stress. Brain Behav. 10, e01525. doi: 10.1002/brb3. 1525

Zhang, X., Dong, H., Li, N., Zhang, S., Sun, J., Zhang, S., et al. (2016). Activated brain mast cells contribute to postoperative cognitive dysfunction by evoking microglia activation and neuronal apoptosis. J. Neuroinflamm. 13:127. doi: 10.1186/s12974-016-0592-9
Zhou, R., Wang, J., Han, X., Ma, B., Yuan, H., and Song, Y. (2019). Baicalin regulates the dopamine system to control the core symptoms of ADHD. Mol. Brain 12:11. doi: 10.1186/s13041-019-0428-5

Conflict of Interest: The authors declare that the research was conducted in the absence of any commercial or financial relationships that could be construed as a potential conflict of interest.

Copyright (c) $2020 \mathrm{Li}$, Su, Cai, Cao, Miao, Zang, Gao, Xu, Yang, Tao and Ai. This is an open-access article distributed under the terms of the Creative Commons Attribution License (CC BY). The use, distribution or reproduction in other forums is permitted, provided the original author(s) and the copyright owner(s) are credited and that the original publication in this journal is cited, in accordance with accepted academic practice. No use, distribution or reproduction is permitted which does not comply with these terms. 